\title{
MHD Simulations of the Collapsar Model for GRBs
}

\author{
Daniel Proga*, Andrew I. MacFadyen ${ }^{\dagger}$, Philip J. Armitage* and Mitchell \\ C. Begelman* \\ * JILA, University of Colorado, Boulder, CO 80309-0440, USA \\ ${ }^{\dagger}$ California Institute of Technology, Mail Code 130-33, Pasadena, CA 9112
}

\begin{abstract}
We present results from axisymmetric, time-dependent magnetohydrodynamic (MHD) simulations of the collapsar model for gamma-ray bursts. Our main conclusion is that, within the collapsar model, MHD effects alone are able to launch, accelerate and sustain a strong polar outflow. We also find that the outflow is Poynting flux-dominated, and note that this provides favorable initial conditions for the subsequent production of a baryon-poor fireball.
\end{abstract}

\section{INTRODUCTION}

The collapsar model is one of most promising scenarios to explain the huge release of energy in a matter of seconds, associated with gamma-ray bursts (GRBs; [1, 2, 3, 4, 5]). In this model, the collapsed iron core of a massive star accretes gas at a high rate $\left(\sim 1 \mathrm{M}_{\odot} \mathrm{s}^{-1}\right)$ producing a large neutrino flux, a powerful outflow, and a GRB. Although the association of long duration GRBs with stellar collapse is now secure $([6,7])$, basic properties of the central engine are uncertain. In part, this is because previous numerical studies of the collapsar model did not explicitly include magnetic fields, although they are commonly accepted as a key element of accretion flows and outflows.

We present a study of the time evolution of 2.5-dimensional, magnetohydrodynamic (MHD) flows in the collapsar model. This study is an extension of existing models of MHD accretion flows onto a black hole (BH; [8]). In particular, we include a realistic equation of state, photodisintegration of bound nuclei and cooling due to neutrino emission. Our study is also an extension of collapsar simulations by [3], as we consider very similar neutrino physics and initial conditions but solve MHD instead of hydrodynamical equations.

\section{MODELS}

We begin the simulations after the $1.7 \mathrm{M}_{\odot}$ iron core of a $25 \mathrm{M}_{\odot}$ presupernova star has collapsed and study the ensuing accretion of the $7 \mathrm{M}_{\odot}$ helium envelope onto the central black hole formed by the collapsed iron core. We consider a spherically symmetric progenitor model, but with spherical symmetry broken by the introduction of a small, latitude-dependent angular momentum and a weak radial magnetic field. For more 
details, see [9]).

\section{RESULTS}

We find that after a transient episode of infall, lasting $0.13 \mathrm{~s}$, the gas with $l \gtrsim 2 R_{S} c$ piles up outside the black hole and forms a thick torus bounded by a centrifugal barrier near the rotation axis. Soon after the torus forms (i.e., within a couple of orbital times at the inner edge), the magnetic field is amplified by the magnetorotational instability (MRI, e.g., [10]) and shear. We have verified that most of the inner torus is unstable to MRI, and that our simulations have enough resolution to resolve, albeit marginally, the fastest growing MRI mode. The torus starts evolving rapidly and accretes onto the black hole. Another important effect of magnetic fields is that the torus produces a magnetized corona and an outflow. The presence of the corona and outflow is essential to the evolution of the inner flow at all times and the entire flow close to the rotational axis during the latter phase of the evolution. We find that the outflow very quickly becomes sufficiently strong to overcome supersonically infalling gas (the radial Mach number in the polar funnel near the inner radius is $\sim 5$ ) and makes its way outward, reaching the outer boundary at $t=0.25 \mathrm{~s}$. Due to limited computing time, our simulations were stopped at $t=0.28215 \mathrm{~s}$, which corresponds to 6705 orbits of the flow near the inner boundary. We expect the accretion to continue much longer, roughly the collapse timescale of the Helium core $(\sim 10 \mathrm{~s})$, as in [3].

Figure 1 shows the flow pattern of the inner part of the flow at $t=0.2437 \mathrm{~s}$. The left and middle panels show density and $\left|B_{\phi}\right|$ maps, respectively. The two maps are overlaid with the direction of the poloidal velocity. The right panel shows the flow domains of different polarity of $B_{\phi}$ overlaid with the direction of the poloidal magnetic field. The polar regions of low density and high $B_{\phi}$ coincide with the region of an outflow. We note that during the latter phase of the evolution not all of the material in the outflow originated in the innermost part of the torus - a part of the outflow is "peeled off" the infalling gas at large radii by the magnetic pressure (see Fig. 2 in [9]). We imposed a weak poloidal field of a given polarity at the outer boundary. This means that initially $B_{\phi}$ changes sign only across the equator, which is a relatively unfavorable configuration for subsequently producing $B_{\phi}$ reversals in the jet. Nevertheless, we find that the polarity of $B_{\phi}$ changes with time. This is because the flow loses memory of the initial polarity by the time it reaches the inner MRI-dominated regions where the jet is formed.

Sikora et al. (2003) [11] argue that Poynting flux-dominated jets with reversing B-fields provide a natural and efficient way to dissipate energy via the reconnection process. Poynting flux dominated jets have been found in previous numerical simulations whereas jets with reversing B-fields appear as a relatively new result. Therefore, we have reviewed results from [8] to check whether polar outflows generated during adiabatic accretion onto BHs also exhibit reversing $B_{\phi}$. Simulations reported by [8] are suitable to study the flow pattern on relatively large length scales because they have been continued for a few dynamical time scales at a distance of $\sim 10^{3} \mathrm{BH}$ radii.

Figure 2 shows the flow domains of different polarity of $B_{\phi}$ overlaid with the direction of the poloidal velocity and magnetic field (left and right panel, respectively) for run D 

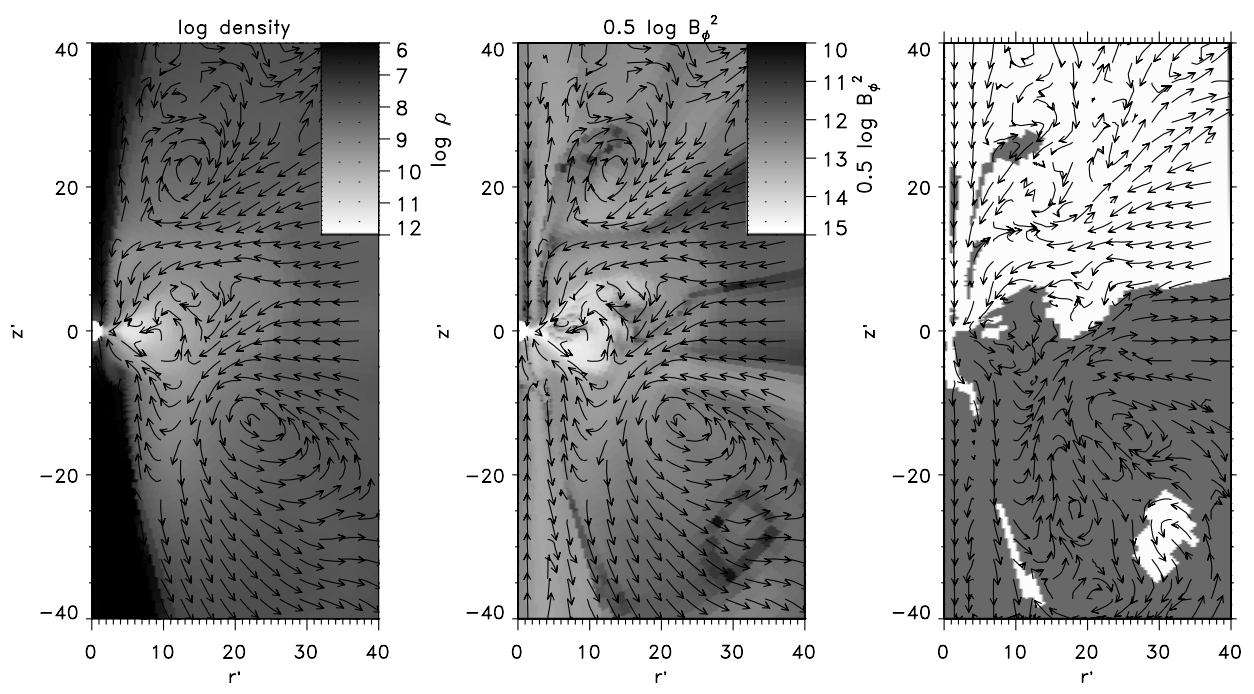

FIGURE 1. Maps of logarithmic density, toroidal magnetic field, and toroidal magnetic field domains with different polarity (white and grey regions) for Proga et al.'s [9] GRB simulation at $t=0.2437 \mathrm{~s}$ (left, middle, and right panel, respectively). The arrows in the left and middle panels indicate the direction of the poloidal velocity while the arrows in right panel indicate the direction of the poloidal magnetic field. The length scale is in units of the $\mathrm{BH}$ radius (i.e., $r^{\prime}=r / R_{S}$ and $z^{\prime}=z / R_{S}$

in [8]. It appears then that reversing B-fields are not unique to our GRB simulations and that domains with different polarity can be relatively large and long-lived.

\section{CONCLUSIONS}

We have performed time-dependent two-dimensional MHD simulations of the collapsar model. Our simulations show that: 1) soon after the rotationally supported torus forms, the magnetic field very quickly starts deviating from purely radial due to MRI and shear. This leads to fast growth of the toroidal magnetic field as field lines wind up due to the torus rotation; 2) The toroidal field dominates over the poloidal field and the gradient of the former drives a polar outflow against supersonically accreting gas through the polar funnel; 3) The polar outflow is Poynting flux-dominated; 4) The polarity of the toroidal field can change with time; 5) The polar outflow reaches the outer boundary of the computational domain $\left(5 \times 10^{8} \mathrm{~cm}\right)$ with an expansion velocity of $\left.0.2 \mathrm{c} ; 6\right)$ The polar outflow is in a form of a relatively narrow jet (when the jet breaks through the outer boundary its half opening angle is $5^{\circ}$ ); 7) Most of the energy released during the accretion is in neutrinos, $L_{v}=2 \times 10^{52} \mathrm{erg} \mathrm{s}^{-1}$. Neutrino driving will increase the outflow energy (e.g., [12] and references therein), but could also increase the mass loading of the outflow if the energy is deposited in the torus. 

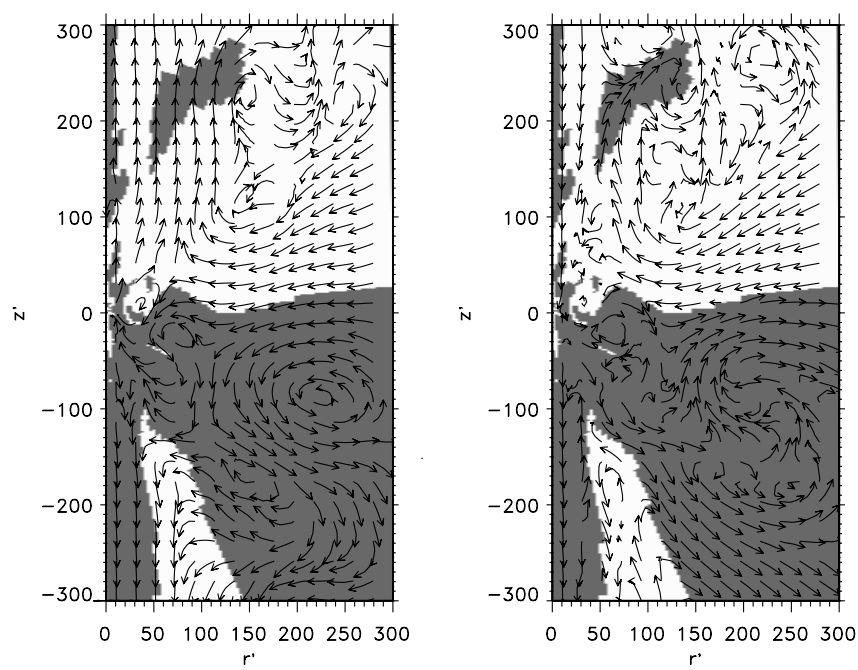

FIGURE 2. Maps of toroidal magnetic field domains with different polarity (white and grey regions) overplotted with the direction of the poloidal velocity (left panel) and the direction of the poloidal magnetic field (right panel) at the end of Proga \& Begelman's simulation of adiabatic accretion onto a $\mathrm{BH}$ (run D in [8]). The length scale is also in units of the BH radius; the same as in Figure 1. Note however the difference in the $r^{\prime}$ and $z^{\prime}$ ranges.

\section{ACKNOWLEDGMENTS}

DP acknowledges support from NASA under LTSA grants NAG5-11736 and NAG512867. MCB acknowledges support from NSF grants AST-9876887 and AST-0307502.

\section{REFERENCES}

1. Woosley, S. E., ApJ 405, 273 (1993).

2. Paczyński, B., ApJ 494, L45 (1998).

3. MacFadyen, A., Woosley, S. E., ApJ 524, 262 (1999).

4. Popham, R., Woosley, S. E., Fryer, C., ApJ 518, 356 (1999).

5. MacFadyen, A., Woosley, S. E., Heger, A., ApJ 550, 410 (2001).

6. Hjorth, J., et al. , Nature 423, 847 (2003).

7. Stanek, K. Z., et al. , ApJ 591, L17 (2003).

8. Proga, D., Begelman, M. C., ApJ 592, 767 (2003).

9. Proga, D., MacFadyen, A. I., Armitage, P. J., Begelman M. C., ApJ 599, L5 (2003).

10. Balbus, S. A., Hawley, J. F., ApJ 376, 214 (1991).

11. Sikora M., Begelman M. C., Coppi P., Proga D., submitted to ApJ Letters (astro-ph/0309504) (2003).

12. Fryer, C. L., Mészáros, P., ApJ 588, L25 (2003). 